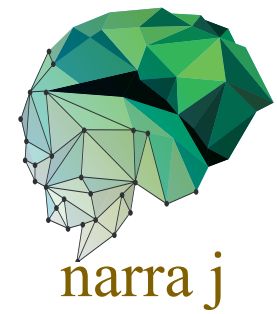

Case Report

\title{
SARS-CoV-2 and Orientia tsutsugamushi co- infection in a young teen, Nepal: Significant burden in limited-resource countries in Asia?
}

\author{
Anup Bastola ${ }^{1, a}$, Ranjit Sah ${ }^{2,3, a *}$, Sagar K. Rajbhandari' ${ }^{1}$ Runa Jha3, Zareena \\ Fathah4, Bimal S. Chalise', Bikesh Shrestha', Rajesh K. Shah', Pujan Balla', Richa \\ Nepal1, Bipin Adhikari5,6, Ali A. Rabaan7, Kuldeep Dhama ${ }^{8}$ and Alfonso J. \\ Rodriguez-Morales9,10
}

${ }^{1}$ Sukraraj Tropical and Infectious Disease Hospital, Teku, Kathmandu, Nepal; ${ }^{2}$ Department of Microbiology, Institute of Medicine, Tribhuvan University Teaching Hospital, Kathmandu, Nepal; 3National Public Health Laboratory, Kathmandu, Nepal; 4King's College London, London, United Kingdom; ${ }^{5}$ Nepal Community Health and Development Centre, Kathmandu, Nepal; ${ }^{6}$ Centre for Tropical Medicine and Global Health, Nuffield Department of Medicine, University of Oxford, Oxford, United Kingdom; 7 Johns Hopkins Aramco Healthcare, Dhahran, Saudi Arabia; ${ }^{8}$ Division of Pathology, ICAR-Indian Veterinary Research Institute, Izatnagar, Uttar Pradesh, India; ${ }^{9}$ Grupo de Investigación Biomedicina, Faculty of Medicine, Fundación Universitaria Autónoma de las Américas, Pereira, Risaralda, Colombia; ${ }^{10}$ School of Medicine, Universidad Privada Franz Tamayo, Cochabamba, Bolivia

aBoth authors contributed equally to this article

*Corresponding authors: ranjitsah57@gmail.com

\section{Abstract}

Scrub typhus is caused by Orientia tsutsugamushi, transmitted through bites of infected chiggers (larval mites). During the coronavirus disease 2019 (COVID-19) pandemic, reports of co-infections with endemic pathogens are increasing around the world. Disease with similar clinical presentation may mask other disease diagnosis and increase the morbidity and mortality of the patients. We report co-infection between severe acute respiratory syndrome coronavirus 2 (SARS-CoV-2) and $O$. tsutsugamushi in a patient in Nepal presenting with fever, headache, retro-orbital pain, generalized body ache, and knee joints pain with a history of dry cough and dyspnea at night. Since scrub typhus is prevalent and considerate as one of the public health consents in Asian countries and the possible overlapping clinical manifestation with other infections including COVID-19, a further investigation required to determine the burden of SARS-CoV-2 and $O$. tsutsugamushi co-infection in scrub typhus-endemic countries in Asia.

Keywords: Scrub typhus, Orientia tsutsugamushi, SARS-CoV-2, COVID-19, coinfection

\section{Introduction}

Coronavirus disease 2019 (COVID-19), caused by severe acute respiratory syndrome coronavirus 2 (SARS-CoV-2), has posed huge concerns while spreading to more than 225 countries affecting nearly 150 million people, with 3.2 million deaths (April 26, 2021). In Nepal, the first case was detected on January 23, 2020, and as of April 26, 2021, a total of 300,119 cases were identified [1]. Overlapping with the ongoing COVID-19 pandemic, Nepal is also facing seasonal vector-borne diseases. Heavy rain and a humid climate provide suitable conditions for disease vectors, including chiggers. The most common vector-borne diseases in Nepal are dengue, malaria, and scrub typhus [2]. Scrub typhus, a disease caused by an obligate 
intracellular bacterium, Orientia tsutsugamushi, is transmitted by chigger mite bites and emerged in Nepal following a massive earthquake in 2015 [3]. Disease appears following rainfall from July with activity reported unti November. In the current scenario, COVID-19 could overlap with one or more vector-borne conditions. We describe a case of co-infection with COVID-19 and scrub typhus in Nepal.

\section{Case}

A fourteen-year-old female resident of Pyutar-4 Lalitpur district, Bagmati province, Nepal, presented at the tertiary care Sujraraj Tropical and infectious Disease Hospital in Lalitpuron on June 26, 2020. The patient complained of a fever for one week with a maximum temperature of $38.8^{\circ} \mathrm{C}$. The illness was associated with headache, retro-orbital pain, generalized body ache, and knee joint pain. She also had a history of dry cough and dyspnea at night. She was examined in the outpatient department. On clinical evaluation, her temperature was $38.3^{\circ} \mathrm{C}$. No other relevant clinical findings were found. Laboratory tests were conducted including a SARS-CoV-2 RT-PCR test, from a nasopharyngeal swab, which was positive (Table 1). The patient was admitted for isolation upon the positive SARS-CoV-2 RT-PCR result on June 28, 2020.

On admission, the patient mentioned a history of dry cough and sore throat. The patient had no other chronic diseases nor any habit of substance abuse. The patient has not visited any place following government-imposed lockdown. The patient had four family members who had alsonot visited any other location during the lockdown. Her father, who works in waste management in Kathmandu, visited her while the patient was ill. On clinical examination, there was no pallor, icterus, cyanosis, clubbing and oedema. The body temperature was $36.5^{\circ} \mathrm{C}$, blood pressure was 108/72 mmHg, pulse of $112 \mathrm{bpm}$ and saturation in room air of 95\%. The remaining systemic examination was unremarkable.

Table 1. Clinical laboratory results for the 14-year-old girl with possible SARS-CoV-2 and scrub typhus co-infection, Nepal

\begin{tabular}{|c|c|c|}
\hline Laboratory finding & June 26, 2020 & June 29, 2020 \\
\hline \multicolumn{3}{|l|}{ Blood analysis } \\
\hline Leukocyte count, $\times 10^{9}$ cells $/ \mathrm{L}$ & 3.15 & 6.40 \\
\hline Neutrophil, \% & 46.00 & 27.00 \\
\hline Lymphocytes, \% & 53.00 & 63.00 \\
\hline Monocytes, \% & 1.00 & 7.00 \\
\hline Eosinophils, \% & ND & 3.00 \\
\hline Platelet count $/ \mathrm{mL}$ & 152,000 & 144,000 \\
\hline Hemoglobin level, g/dL & 10.7 & 10.5 \\
\hline Random blood sugar, $\mathrm{mg} / \mathrm{dL}$ & 117 & 140 \\
\hline Creatinine, $\mathrm{mg} / \mathrm{dL}$ & 0.4 & 0.5 \\
\hline $\mathrm{Na}, \mathrm{mmol} / \mathrm{L}$ & 141 & $\mathrm{ND}$ \\
\hline $\mathrm{K}, \mathrm{mEq} / \mathrm{L}$ & 4 & ND \\
\hline C-reactive protein, $\mathrm{mg} / \mathrm{L}$ & 14 & Negative \\
\hline Direct Bilirubin, mg/dL & ND & 1.2 \\
\hline Total Bilirubin, mg/dL & ND & 0.2 \\
\hline ALT, IU/L & ND & 150 \\
\hline AST, IU/L & ND & 161 \\
\hline \multicolumn{3}{|l|}{ Urinalysis } \\
\hline Albumin & Not present & ND \\
\hline Sugar & Not present & ND \\
\hline Cast & Not present & ND \\
\hline Pus cells & 2-3 per field & $\mathrm{ND}$ \\
\hline Red blood cells & $0-2$ per field & ND \\
\hline Real-time RT-PCR for SARS-CoV-2 & Positive & Positive \\
\hline \multicolumn{3}{|l|}{ DENV serological tests } \\
\hline IgM antibodies & ND & Negative \\
\hline IgG antibodies & ND & Negative \\
\hline NS1 Antigen & ND & Negative \\
\hline \multicolumn{3}{|l|}{ Scrub typhus serological tests } \\
\hline IgM antibodies & ND & Positive \\
\hline IgG antibodies & ND & Positive \\
\hline
\end{tabular}

ALT: alanine aminotransferase, AST: aspartate aminotransferase, DENV: dengue virus, MAT: microscopic agglutination test, ND: not determined, RT-PCR: reverse transcription PCR. 
Follow-up laboratory investigations on June 29, 2020 suggested leukocytopenia, thrombocytopenia and elevated transaminases. As these signs are commonly seen in scrub typhus infection and are less common in milder forms of COVID-19 infection, scrub typhus was investigated. A rapid diagnostic test for scrub typhus (Diagnostic NIC, Vancouver, Canada) returned positive for IgG and IgM antibodies against $O$. tsutsugamushi. Serological tests for dengue (Diagnostic NIC, Vancouver, Canada) were negative (Table 1).

The chest X-rays and computed tomography (CT)-scan of the patient were normal, except for the finding of dextrocardia, giving the idea of lack of evolution of severe COVID-19 manifestations. Investigation of patient's residential area found a landscape with vegetation, serving as a suitable habitat for both mice and chiggers.

\section{Discussion}

COVID-19 is an ongoing pandemic affecting nations worldwide [5]. COVID-19 presents with many non-specific symptoms such as fever (83-99\%), cough (59-82\%), fatigue (44-70\%), anorexia (40-84\%), shortness of breath (31-40\%) and myalgias (11-35\%), that may overlap with vector-borne diseases, such as dengue and scrub typhus [5-8]. There are other less common symptoms which have also been reported such as sore throat, nasal congestion, headache, diarrhea, nausea and vomiting. Anosmia and ageusia have also been described in high frequency $[7,9]$. The reported case presented with fever, headache, retro-orbital pain, generalized myalgia, joint pain, cough, dyspnea and sore throat. Symptoms presented in this case overlapped both with the COVID-19 and scrub typhus. Severe headache, myalgia, retroorbital pain are common with scrub typhus, and cough and dyspnea are seen among COVID-19 patients. No cases of COVID-19 and scrub typhus have been reported in the literature.

This case highlights the importance of differential diagnoses in endemic areas with conditions such as scrub typhus and the possibility of co-infection with this and other tropical pathogens [10]. In coming days, these incidences may rise as scrub typhus activity approaches peak activity from August, coinciding with continual COVID-19 spread within the community, making it difficult for clinicians to recognize and differentiate these two diseases. The ongoing COVID-19 pandemic and continual spread within the country, along with other vector-borne conditions, including scrub typhus, may lead to difficulties for clinicians to make precise diagnoses. Clinicians must also consider and evaluate other vector-borne diseases along with COVID-19, including scrub typhus, in endemic areas, or travelers coming from the endemic areas.

Scrub typhus is considered one of the emerging public health issues in Asian countries [11]. Although scrub typhus is mainly limited to the Asia Pacific region, one million cases are reported annually, and nearly one billion people are at risk of getting infected [12]. Based on overlapping clinical manifestations, it is possible that the occurrence of scrub typhus is overshadowed by COVID-19 in several Asian countries where the disease is endemic, especially in Nepal [13]. COVID-19 closely mimics other diseases having acute febrile illnesses such as dengue fever, scrub typhus, leptospirosis, and malaria [11,14]. The rapid spread of SARS CoV-2 infections in several tropical countries has resulted in a diagnostic challenge for detecting acute febrile illnesses. To differentiate scrub typhus from COVID-19, clinicians can investigate the presence of eschar, a classic pathognomonic sign, and the most vital indicator for diagnosing scrub typhus [14].

\section{Conclusions}

We reported a possible COVID-19 and scrub typhus co-infection in a young teen in Nepal. Although the patient was diagnosed for scrub typhus by a rapid diagnostic test with single spot reading only, this highlights the important for the clinicians to evaluate the possible coinfections of COVID-19 with other endemic tropical infections in particular in limited-resource countries in Asia. 


\section{Declarations}

\section{Ethics approval}

Not applicable for case report.

\section{Patient's consent}

Informed written consent was taken from the patient to publish this case report and related evidence.

\section{Acknowledgments}

Thanks to the patient for giving consent to publish this case and the tertiary care center for referring the case.

\section{Conflict of interest}

Authors declare no conflict of interest.

\section{Funding}

This study received no funding.

\section{How to cite}

Bastola A, Sah R, Rajbhandari SK, et al. SARS-CoV-2 and Orientia tsutsugamushi co-infection in a young teen, Nepal: Significant burden in limited-resource countries in Asia? Narra J 2021; 1(2):e34. http://doi.org/10.52225/narraj.v1i2.34.

\section{References}

1. Health Emergency Operation Center, Ministry of Health and Population, Government of Nepal. Coronavirus disease (COVID-19) outbreak updates \& resource materials. https://heoc.mohp.gov.np/update-on-novel-corona-viruscovid-19/ (Accessed: 26 June 2021).

2. Epidemiology and Disease Control Division, Ministry of Health and Population, Government of Nepal. Vector-Borne Disease Updates. http://www.edcd.gov.np/news/dengue-updates1 (Accessed: 26 June 2021).

3. Bastola APN. An outbreak of scrub typhus in Nepal following the 2015 Gorkha earthquake. Int J Infect Dis 2016; 53:55.

4. Kim DM, Lee YM, Back JH, et al. A serosurvey of Orientia tsutsugamushi from patients with scrub typhus. Clin Microbiol Infect. 2010;16(5):447-451.

5. Dhama K, Khan S, Tiwari R, et al. Coronavirus Disease 2019-COVID-19. Clin Microbiol Rev. 2020;33(4):e00028-20.

6. Bastola A, Sah R, Rodriguez-Morales AJ, et al. The first 2019 novel coronavirus case in Nepal. Lancet Infect Dis 2020; 20(3):279-280.

7. Ollarves-Carrero MF, Rodriguez-Morales AG, Bonilla-Aldana DK, Rodríguez-Morales AJ. Anosmia in a Healthcare Worker with COVID-19 in Madrid, Spain. Travel Med Infect Dis 2020; 35:101666.

8. Cardona-Ospina JA, Arteaga-Livias K, Villamil-Gómez WE, et al. Dengue and COVID-19, overlapping epidemics? An analysis from Colombia. J Med Virol. 2020: 93(1):522-527.

9. Vargas-Gandica J, Winter D, Schnippe R, et al. Ageusia and anosmia, a common sign of COVID-19? A case series from four countries. J Neurovirol. 2020;26(5):785-789.

10. Wilder-Smith A. COVID-19 in comparison with other emerging viral diseases: risk of geographic spread via travel. Trop Dis Travel Med Vaccines. 2021;7(1):3.

11. Musa TH, Ahmad T, Wana MN, et al. The epidemiology, diagnosis and management of scrub typhus disease in China. Hum Vaccin Immunother. 2021 Jun 14:1-11. doi: 10.1080/21645515.2021.1934355. In press.

12. Xu G, Walker DH, Jupiter D, et al. A review of the global epidemiology of scrub typhus. PLoS Negl Trop Dis. 2017;11(11):e0006062. 
Bastola et al. Narra J 2021; 1 (2): e34 - http://doi.org/10.52225/narraj.v1i2.34

13. Lamsal M. The risk of dengue and scrub typhus overshadowed by COVID-19 in Nepal. Asia Pac J Public Health. 2021 May 3:10105395211012907. doi: 10.1177/10105395211012907. In press.

14. Hazra D, Abhilash KP, Gunasekharan K, et al. An indispensable clue for the diagnosis of scrub typhus and COVID-19 co-infection during the ongoing pandemic. J Postgrad Med. 2021;67(2):117-118. 